Valsalva, we are concerned about the progression of aortic regurgitation and the patient must be kept under close long-term observation.

\section{References}

1. Heilman KJ 3rd, Groves BM, Campbell D, et al. Rupture of left sinus of Valsalva aneurysm into the pulmonary artery. J Am Coll Cardiol. 1985;5:1005-7.
2. Luckraz H, Naik M, Jenkins G, et al. Repair of a sinus of Valsalva aneurysm that had ruptured into the pulmonary artery. J Thorac Cardiovasc Surg. 2004;127: 1823-5.

3. Kar AK, Bhattacharya S, Ray D, et al. Rupture of the sinus of Valsalva into the pulmonary artery. Indian Heart J. 2002;54:415-7

4. Fazio G, Zito R, Dioco DD, et al. Rupture of a left sinus of Valsalva aneurysm into the pulmonary artery. Eur J Echocardiogr. 2006;7:230-2; Epub 2006 Jan 6.

5. Shiraishi S, Watarida S, Katsuyama K, et al. Unruptured aneurysm of the sinus of Valsalva into the pulmonary artery. Ann Thorac Surg. 1998;65:1458-9.

\title{
Mechanical support for acute right ventricular failure: Evolving surgical paradigms
}

\author{
Thao T. Marquez, MD, ${ }^{\mathrm{a}}$ Jonathan D’Cunha, MD, PhD, ${ }^{\mathrm{b}}$ Ranjit John, MD, ${ }^{\mathrm{a}}$ Kenneth Liao, MD, ${ }^{\mathrm{a}}$ and Lyle Joyce, MD, PhD, ${ }^{\mathrm{a}}$
} Minneapolis, Minn

Isolated right ventricular (RV) infarction after dissection of the right coronary artery is associated with a high risk of death because of the development of RV failure, cardiogenic shock, and multisystem organ failure (MSOF). There are few reports of the use of RV assist devices (RVADs) for acute RV failure. We describe the successful care of 2 such moribund patients who experienced striking clinical improvement with the RVAD as a novel approach to acute surgical management.

\section{CLINICAL SUMMARY}

PATiEnt 1. A 76-year-old man was evaluated urgently after dissection of the right coronary ostium with extension into his right coronary artery during coronary angioplasty. He was in cardiogenic shock and required the assistance of an intraaortic balloon pump. Angiography showed bilateral coronary artery disease (left anterior descending artery and right main coronary artery). He underwent 2-vessel coronary artery bypass grafting, oversewing of his right coronary ostium, and complete transection of the aorta at the sinotubular ridge with reanastomosis to prevent distal dissection.

Postoperatively, his cardiac dysfunction continued with ventricular fibrillation and cardiac arrest. Reexploration revealed patent grafts; however, echocardiography demonstrated a nonfunctioning right ventricle. We therefore placed a Biomedicus (Eden Prairie, Minn) RVAD and converted to a Levitronix (Waltham, Mass) RVAD centrifu-

\footnotetext{
$\overline{\text { From the Division of Cardiothoracic Surgery }}{ }^{\mathrm{a}}$ and Division of Thoracic and Foregut Surgery, ${ }^{b}$ Department of Surgery, University of Minnesota, Minneapolis, Minn. Received for publication Jan 3, 2008; accepted for publication Feb 23, 2008. Address for reprints: Thao T. Marquez, MD, MMC 450, 420 Delaware St SE, Minneapolis, MN 55113 (E-mail: tran0360@umn.edu).

J Thorac Cardiovasc Surg 2009;137:e39-40

$0022-5223 / \$ 36.00$

Copyright (c) 2009 by The American Association for Thoracic Surgery doi:10.1016/j.jtcvs.2008.02.025
}

gal pump 48 hours later, observing immediate improvement in his systemic pressures. He was weaned from pressors but exhibited evidence of MSOF (oliguria and respiratory failure).

After 11 days of mechanical support, the patient's condition significantly improved. After treatment of a Pseudomonas aeruginosa pneumonia, he was weaned and extubated. His renal insufficiency resolved without intervention. The RVAD was turned down to a flow of 2 liters per minute without any change in hemodynamic parameters. Echocardiography showed full functional recovery of the RV, and the RVAD was explanted. He was discharged on postoperative day 21 , and his condition returned to the functional baseline.

PATIEnT 2. A 36-year-woman was seen at an outside hospital and found to have a type A acute ascending aortic dissection with evidence of RV infarction. She underwent an aortic root replacement with a Medtronic (Minneapolis, Minn) freestyle valve conduit and coronary artery reimplantation. After repair, echocardiography showed severely compromised RV function, and an AbioMed (Danvers, Mass) RVAD was implanted. She had MSOF with liver dysfunction, kidney failure, and refractory hypoxia requiring placement of an extracorporeal membrane oxygenator. We exchanged her temporary AbioMed RVAD to a longerduration Levitronix RVAD that is routinely used at our institution. ${ }^{1}$

Despite a tenuous postoperative course, the patient's hypoxia improved and the extracorporeal membrane oxygenator was weaned on postoperative day 2 . On postoperative day 18, an RVAD turndown echocardiogram showed persistently poor RV function. Coronary angiography revealed an occluded right coronary vascular graft, for which she underwent off-pump single-vessel coronary artery bypass grafting. A repeat intraoperative RVAD turndown echocardiogram resulted in hemodynamic deterioration 
demonstrating intolerance of RVAD removal. We therefore exchanged her paracorporeal Levitronix RVAD for a Thoratec intracorporeal VAD (Pleasanton, Calif). ${ }^{2}$ Postoperatively, she recovered from MSOF and was discharged from the hospital on postoperative day 53.

Repeat RVAD turndown 4 months later showed functional RV recovery, and the patient's Thoratec RVAD was successfully explanted. One month later, she was at home, doing well, and enjoying normal daily activities.

\section{DISCUSSION}

RV failure from acute RV infarction is associated with high morbidity and mortality rates. Early revascularization and RVAD implantation as a bridge to recovery allowed our patients the time and opportunity for RV recovery.

Experience with the use of RVADs to treat isolated RV failure from acute myocardial infarction is limited. RV failure requiring RVAD placement has been a notable complication after LVAD placement. Isolated RV failure in postcardiotomy patients has been described in few studies, suggesting that RV function in this setting is salvageable with early mechanical support. ${ }^{3}$ Longer duration of RVAD support is becoming more feasible as more RVADs are designed to be implantable and portable, such as the Thoratec intracorporeal VAD. This device allowed patient 2 to continue rehabilitation on an outpatient basis, improving her overall quality of life.

Weaning protocols for VADs are varied and yet to be standardized. $^{4,5}$ Our protocol involves echocardiography during an RVAD turndown, and if hemodynamic parameters are maintained, we elect to explant the RVAD. In our experience, hemodynamic stability during RVAD turndown reliably predicts explantation success.

\section{CONCLUSIONS}

RVADs can be used effectively in patients who experience isolated RV infarction secondary to dissection of the right coronary artery. Our experience suggests that patients may successfully undergo RVAD placement as a bridge to recovery or heart transplantation. RVADs should be considered early in the care of patients with isolated RV failure as a result of acute myocardial infarction.

We thank Mary E. Knatterud, $\mathrm{PhD}$, for editorial assistance.

\section{References}

1. John R, Liao K, Leitz K, Kamdar F, Colvin-Adams M, Boyle A. Experience with the Levitronix CentriMag circulatory support system as a bridge to decision in patients with refractory acute cardiogenic shock and multisystem organ failure. J Thorac Cardiovasc Surg. 2007;134:351-8.

2. Farrar D, Reichenbach SH, Rossi SA, Weidman JR. Development of an intracorporeal Thoratec ventricular assist device for univentricular or biventricular support. ASIAO J. 2000;46:351-3.

3. Moazami N, Pasque M, Moon M. Mechanical support for isolated right ventricular failure in patients after cardiotomy. J Heart Lung Transpl. 2004;23: 1371-5.

4. Delgado D, Rao V, Miriuka S. Explantation of a mechanical assist device: assessment of myocardial recovery. J Card Surg. 2004;17:47-50.

5. Slaughter M, Sobieski M, Koening S, Pappas P, Tatooles A, Silver M. Left ventricular device weaning: hemodynamic response and relationship to stroke volume and rate reduction protocols. ASIAO J. 2006;52:228-33.

\title{
Successful cardiac transplant after Berlin Heart bridge in a single ventricle heart: Use of aortopulmonary shunt as a supplementary source of pulmonary blood flow
}

\author{
F. Bennett Pearce, MD, ${ }^{\mathrm{a}}$ James K. Kirklin, MD, ${ }^{\mathrm{b}}$ William L. Holman, MD, ${ }^{\mathrm{b}}$ Cindy S. Barrett, MD, ${ }^{\mathrm{a}}$ Robb L. Romp, MD, ${ }^{\mathrm{a}}$ \\ and Yung R. Lau, MD, ${ }^{\mathrm{a}}$ Birmingham, Ala
}

Pulsatile ventricular assist devices, and in particular the Berlin Heart Excor infant ventricular assist device (Berlin Heart; Berlin Heart AG, Berlin, Germany), have rarely been used to support infants and small children with single

From the Divisions of Pediatric Cardiology ${ }^{\mathrm{a}}$ and Cardiothoracic Surgery, ${ }^{\mathrm{b}}$ UAB School of Medicine, Birmingham, Ala.

Received for publication Jan 24, 2008; accepted for publication Feb 7, 2008.

Address for reprints: F. Bennett Pearce, MD, Division of Pediatric Cardiology, NHB

320, 619 19th St South, Birmingham, AL 35249-6852 (E-mail: pearce@uab.edu). J Thorac Cardiovasc Surg 2009;137:e40-2

$0022-5223 / \$ 36.00$

Copyright (C) 2009 by The American Association for Thoracic Surgery doi:10.1016/j.jtcvs.2008.02.044 ventricle. $^{1,2}$ We report successful cardiac transplantation in an infant with single ventricle supported with a Berlin Heart.

\section{CLINICAL SUMMARY}

A 15-month-old boy with the diagnosis of $\{$ S,D,D $\}$ double-outlet right ventricle, mitral valve atresia, D-malposition of the great vessels, status-post pulmonary artery band in infancy, and poor systemic ventricular function was referred for transplant evaluation. A catheterization performed at the referral institution 3 months before admission documented mean central venous pressure of $25 \mathrm{~mm} \mathrm{Hg}$, poor 